\title{
Face Recognition with LWIR Imagery Using Local Binary Patterns
}

\author{
Heydi Méndez ${ }^{1}$, Cesar San Martín ${ }^{2}$, Josef Kittler ${ }^{3}$, Yenisel Plasencia ${ }^{1}$, \\ and Edel García-Reyes ${ }^{1}$ \\ 1 Advanced Technologies Application Center, \\ 7a \# 21812 e/ 218 y 222, Rpto. Siboney, Playa, C.P. 12200, La Habana, Cuba \\ $\{$ hmendez, ylpasencia, egarcia\}@cenatav.co.cu \\ 2 Dep. of Electrical Eng. University of La Frontera, Casilla 54-D, Temuco, Chile \\ csmarti@ufro.cl \\ 3 University of Surrey, Guildford, Surrey, GU2 7XH, UK \\ J.Kittler@surrey.ac.uk
}

\begin{abstract}
In this paper, the merits of the Local Binary Patterns (LBP) representation are investigated in the context of face recognition using long-wave infrared images. Long-wave infrared images are invariant to illumination, but at the same time they are affected by a fixed-pattern noise inherent to this technology. The fixed-pattern is normally compensated by means of a non-uniformity correction method. Our study shows that the LBP approach is robust to the fixed-pattern noise, as well as to the presence of glasses. Not only no noise suppressing preprocessing is needed, but in fact if a non-uniformity correction method is applied, the image texture is amplified and the performance of the LBP degraded.
\end{abstract}

\section{Introduction}

The sensitivity to variations in illumination is one of the major limiting factors for face recognition system performance. Different methods have been proposed in the literature aiming to compensate for illumination variations, but the classification performance is still affected [1. Among them, infrared (IR) imaging has been shown to be less affected by changes in ambient illumination. IR spectrum typically is divided in two groups: reflected IR $(0.7-2.4 \mu \mathrm{m})$ and thermal IR $(2.4 \mu \mathrm{m}-14 \mathrm{~mm})$. Near-IR belongs to the reflected IR group. Being reflected by objects, it can serve as an active illumination source. The merits of using active Near-IR to provide constant illumination for face recognition have been demonstrated [2]3]. On the other hand, long-wave IR (LWIR), which belongs to the thermal IR, presents the following advantages: i) LWIR sensors collect the heat energy emitted by a body instead the light reflected; ii) it also exhibits invariant behavior under changes in illumination, being able to operate even in complete darkness; and iii) human skin has a high emissivity in $8-12 \mu \mathrm{m}$ presenting a thermal signature which is unique to each individual.

In this paper, we focus on LWIR face recognition because of its benefits for outdoor applications where the environment illumination conditions are too variable. In the context of the previous work on face recognition using LWIR imagery 
[45], some papers compare LWIR with visible images [6]7 and fuse these two kinds of data 89, showing promising results. In most of the cases, appearance based methods are used to represent and recognize LWIR images [7]0. However, face recognition using LWIR imagery with Local Binary Patterns (LBP) has received little attention in the literature [9], in spite of its simplicity of implementation, efficiency and robustness already demonstrated in visible and Near-IR face recognition tasks.

An IR system consists of a group of detectors placed in an array located at the focal plane of an imaging optical system, known as focal-plane array (FPA) technology [1]. FPA is hindered by the non-uniformity (NU) effect, manifest in fixed-pattern noise (FPN), i.e. a pixel-to-pixel variation in the sensor array, caused by the differences between semiconductors. The FPN is superimposed on the true images degrading the quality of the captured data [12]. In order to solve this problem, several NU correction methods have been proposed to compensate for the NU noise in IR-FPA. For example, image-registration-based algorithms 13, algebraic scene-based algorithms [14, the constant statistics algorithms [15], and Kalman-filter-based algorithms [16 17], among others. But, to our knowledge, only in 18, these compensation methods are applied to LWIR face recognition, improving significantly the recognition performance.

In this paper we evaluate the performance of the LBP method applied to LWIR face recognition and its response to FPN. We show that LBP is very robust to the FPN and surprisingly its discriminatory capacity is degraded when the image texture is affected by random noise. In Section 2, we review the LBP method and discuss the origins, nature and effect of noise in IR images. Section 3 evaluates the performance of the proposed methodology. Section 4 concludes the paper.

\section{Methodology}

In this section the LBP method is described and basic notions about noise in LWIR images are presented.

\subsection{Local Binary Patterns}

The use of the LBP operator in face recognition was introduced in [19] and different extensions of the original operator have appeared afterwards [20]. As it can be appreciated in Figure 1, the original LBP operator represents each pixel of an image by thresholding its $3 x 3$ - neighbourhood with reference to the center pixel value, $g_{c}$, and considering the result as a binary number, called the LBP code. The image is then divided into rectangular regions and histograms of the LBP codes are calculated over each of them. Finally, the histograms of each region are concatenated into a single one that represents the face image. A dissimilarity measure is used to compare the histograms of two different images. 


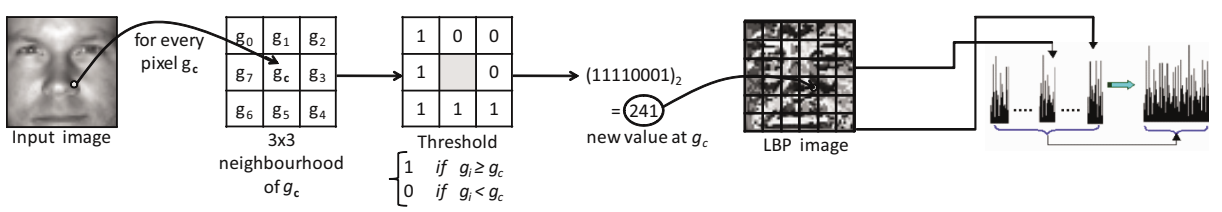

Fig. 1. Local Binary Patterns

\subsection{Non-Uniformity Noise in LWIR Images and Its Correction}

The IR detector has several known sources of noise, but the main contributing factors to signal corruption are the FPA temporal noise, the FPA NU noise, and the readout noise injected by the output electronics 12 . The FPN refers to any spatial pattern that does not change significantly from frame-to-frame, and it is due to differences in detector size, doping density and foreign matter getting trapped during fabrication. The multiplicative component of the FPN is due to the FPA photoresponse NU, which means that the responsivity changes from one detector to another. This component is produced by pixel-to-pixel differences in responsivity or gain, the detector size, spectral response and thickness in coatings of each detector. The additive component of the FPN, white noise, is mainly caused by the dark current, which is kept almost constant and does not vary from frame to frame because of its dependence on the temperature and the offset voltages of the amplifiers used. Taking all the noise process into consideration, each pixel on the FPA can be modelled at instant $n$, using a two-parameter linear equation given by:

$$
Y_{i j}(n)=A_{i j} X_{i j}(n)+B_{i j}+v_{i j}(n),
$$

where $A_{i j}$ and $B_{i j}$ are, respectively, the gain (multiplicative NU) and the offset (additive NU) associated with the $i j t h$ detector. The random process $X_{i j}(n)$ is the IR photon flux collected at the $i j t h$ detector. The term, $v_{i j}(n)$, corresponds to the additive noise, FPA temporal noise, which is commonly modelled as a zero mean white Gaussian random process called temporal random noise. $Y_{i j}(n)$ is referred to as readout data from the IR-FPA system.

In order to solve this problem, several NU compensation techniques have been developed. They can be divided into calibration techniques and scenebased correction methods. The first group requires two uniform references from blackbody radiator at different temperatures, and then, the gain and offset are obtained. The NU compensation is performed using the following equation:

$$
\hat{X}_{i j}(n)=\frac{Y_{i j}(n)-\hat{B}_{i j}}{\hat{A}_{i j}},
$$

where $\hat{A}_{i j}$ and $\hat{B}_{i j}$ are the estimates of the gain and offset, and $\hat{X}_{i j}(n)$ is the estimated IR input irradiance.

The scene-based methods estimate gain and offset, but their performance is limited by the amount of spatio-temporal information and the diversity of 
temperature in the image sequence. The principal limitations of the scene-based method are that the camera must be in constant motion, and that the estimation requires a large number of frames.

A well-known scene-based NUC method is the constant statistics method proposed in [15. The principal assumption of this method is that the input irradiance in mean and variances is the same for all sensors of the FPA. Applying the mean and variance to equation (1), and assuming that the mean and standard deviation of $X_{n}$ are 0 and 1, respectively, the gain and offset can be obtained from:

$$
\begin{gathered}
\hat{A}_{i j}=\sigma_{Y_{i j}}-\sigma_{v_{i j}}, \\
\hat{B}_{i j}=\mu_{Y_{i j}} .
\end{gathered}
$$

In order to obtain a solution with low estimation error, a good method of estimating of the mean and variance (large number of frames)is required. To avoid this conditions we assume that the readout data have a uniform distribution in a known range $\left[Y^{\mathrm{min}}, Y^{\mathrm{max}}\right]$. In such a case the mean and variance can be obtained respectively from:

$$
\mu_{Y_{i j}}=\frac{Y_{i j}^{\max }+Y_{i j}^{\min }}{2} \wedge \sigma_{Y_{i j}}=\frac{Y_{i j}^{\max }-Y_{i j}^{\min }}{\sqrt{12}},
$$

and then, the correction is performed using equation (2) with the values obtained from (3) and (4).

\section{Experimental Evaluation and Discussion}

In our experiments we used the long-wave infrared images collected by Equinox Corporation 21. This database is composed of three 40 frame sequences from 91 persons, acquired in two days with three different light sources: frontal, left lateral and right lateral. The frame sequences were recorded while people were uttering vowels standing in a frontal pose, and three more images from each person were taken to capture the expression of, respectively to smile, frown and surprise. In addition, the complete process was repeated for those persons who wore glasses. Since much of the data of one person is highly correlated, usually only a subset of the images is used for experimentation [6]7.

The LWIR images of Equinox are of $240 x 320$ pixels size, they were radiometrically calibrated and represented as gray-scale images with 12 bits per pixels. The calibrated images from the database present a dead-pixel distortion besides the typical FPN. This source of noise means that the detector always gives the same readout value independent of the input irradiance. In order to remove it, it is possible to assume that the IR irradiance collected by the sensor $i j$ is to be close to the neighbors around the sensor $i j$, then we assumed this value as the readout data.

The different face images of a person are not aligned as can be appreciated in Figure 2, however the displacement is not too great. Taking into account this 

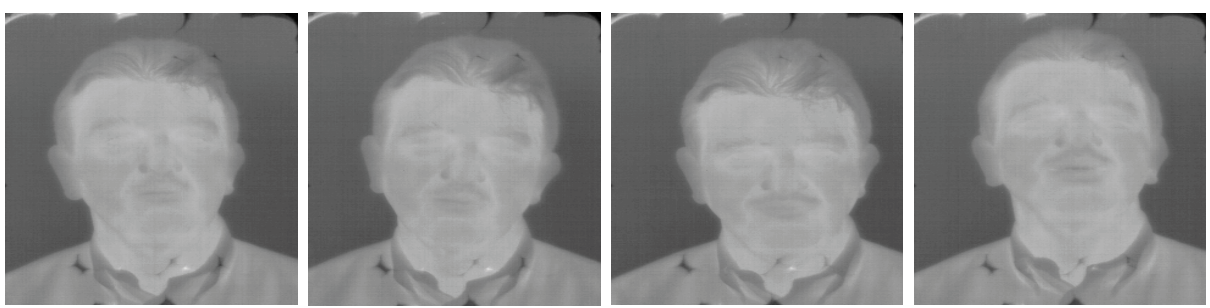

Fig. 2. Examples of LWIR images of one person

fact and that it is not easy to precisely detect the facial features in LWIR, the images were not geometrically normalized. On the other hand, the size of the LBP regions was selected not too small neither too big, $18 x 21$ pixels as in [19].

Because the use of glasses changes the thermal emission perceived in LWIR face images, we split our experiments into two cases: with and without glasses.

\subsection{The No Glasses Case}

In this case, we use the same procedure as other authors [7/8] who constructed the following multiple subsets using only three images of the vocal pronunciation frame sequence (vowel frames) and the three expression frames of each subject in each illumination condition:

VA: Vowel frames, all illuminations.

EA: Expression frames, all illuminations.

VF: Vowel frames, frontal illumination.

EF: Expression frames, frontal illumination.

VL: Vowel frames, lateral illuminations.

EL: Expression frames, lateral illuminations.

The performance of the LBP based matching is evaluated by using each time one set as gallery and another as a test set. Some of the subset combinations are not considered in the experiments since one subset is included in the other. Table 1 below, where the columns represent the gallery sets and the rows the test sets, shows the top-match recognition performance of the LBP method.

Table 1. Correct classification percent with LBP method

\begin{tabular}{|c|c|c|c|c|c|c|}
\hline & VA & EA & VF & EF & VL & EL \\
\hline VA & & 98.13 & 97.75 & 98.50 & 99.25 & 95.13 \\
\hline EA & 97.67 & & 93.77 & 98.44 & 97.67 & 100.0 \\
\hline VF & & 98.13 & & 97.00 & 97.38 & 95.51 \\
\hline EF & 99.24 & & 99.62 & & 99.24 & 95.08 \\
\hline VL & & 98.13 & 96.63 & 96.63 & & 96.63 \\
\hline EL & 97.74 & & 92.83 & 95.09 & 98.49 & \\
\hline
\end{tabular}


Although the alignment of the faces in Equinox images is very similar, even a small localization error usually affects the appearance based methods. The obtained results, giving an average of 97.3 percent of correct classification are very promising, taking into account that images were not geometrically normalised or cropped. This performance is comparable with the best performance of an appearance based method obtained earlier with the same LWIR images previously geometrically transformed, by means of the Linear Discriminant Analysis (LDA) method [22, reported in [7] and summarized in Table 2.

Table 2. Classification results obtained with the LDA method 7]

\begin{tabular}{|l|l|l|l|l|l|l|}
\hline & VA & EA & VF & EF & VL & EL \\
\hline VA & & 99.6 & 98.3 & 96.2 & 99.6 & 99.3 \\
\hline EA & 97.4 & & 94.0 & 98.1 & 96.8 & 99.2 \\
\hline VF & & 100.0 & & 97.0 & 98.8 & 98.6 \\
\hline EF & 97.2 & & 94.6 & & 95.6 & 97.9 \\
\hline VL & & 99.5 & 97.4 & 95.8 & & 99.6 \\
\hline EL & 97.4 & & 93.7 & 97.1 & 97.4 & \\
\hline
\end{tabular}

Note that the results are very similar, with the LDA achieving an average of 97.5 percent of correct classification. However, besides the fact that no geometric normalisation was needed, LBP has the advantage over the LDA method that it does not need more than one image per person in the gallery set, neither it requires a training set, which are very important properties of a face recognition system.

In order to improve the results and following the idea in [18, prior to computing the LBP representation we applied a NU correction method, estimating the FPN as was described in section 2.2 since the blackbody images are not available from 21. Table 3 shows the identification rates obtained when the correction was applied.

Table 3. Classification results with the LBP method after NU correction

\begin{tabular}{|c|c|c|c|c|c|c|}
\hline & VA & EA & VF & EF & VL & EL \\
\hline VA & & 88.89 & 95.63 & 87.89 & 95.38 & 90.14 \\
\hline EA & 97.67 & & 94.94 & 93.77 & 98.05 & 97.67 \\
\hline VF & & 85.77 & & 92.51 & 90.26 & 86.02 \\
\hline EF & 98.48 & & 98.86 & & 98.48 & 94.70 \\
\hline VL & & 91.26 & 93.63 & 83.65 & & 90.76 \\
\hline EL & 96.60 & & 93.96 & 93.21 & 98.11 & \\
\hline
\end{tabular}

Surprisingly, with an average value of 93.3 percent, the performance with the NU correction was lower than without it. Inspecting the original and NU corrected images in Figure 3, it is apparent that although the NUC method 

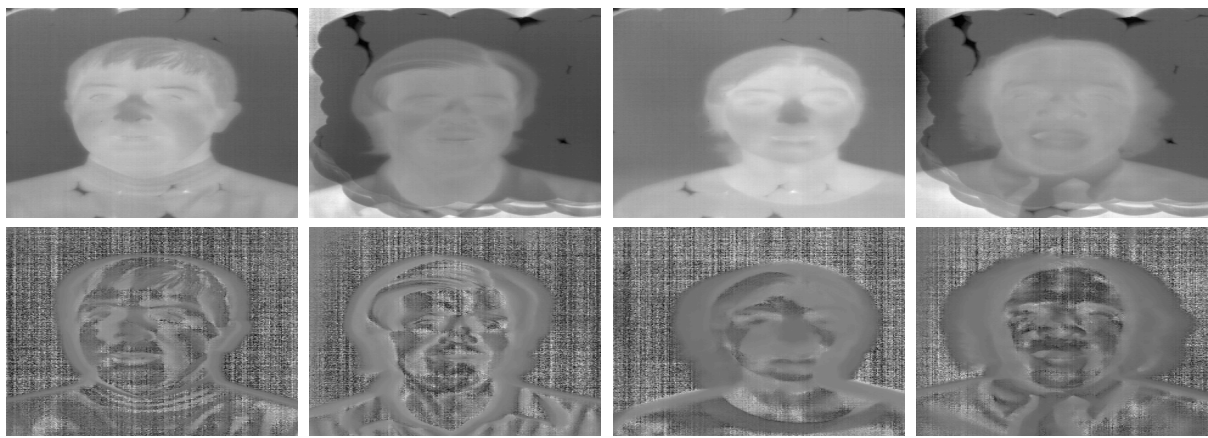

Fig. 3. Examples of LWIR original (first row) and NU corrected (second row) images

suppresses fixed-pattern noise in the IR images, the random noise is magnified and the image texture is affected. Since LBP is a texture descriptor, it is sensitive to this kind of noise.

In order to support the hypothesis that the LBP method is sensitive to random noise in LWIR images, we conducted the same experiments adding some random noise artificially to the original images, as can be seen in Figure 4.
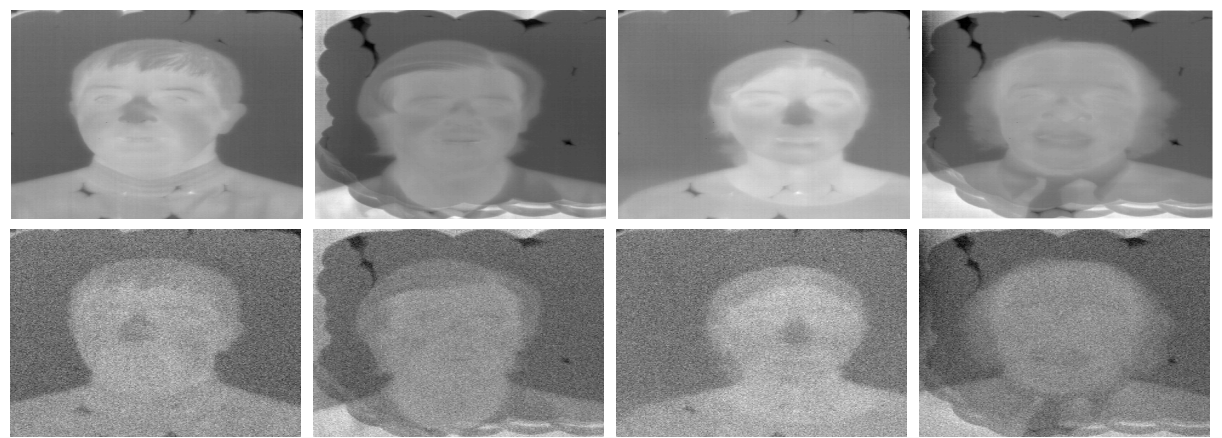

Fig. 4. Examples of LWIR original (first row) and random noise affected (second row) images

Table 4 displays the results of the experiments adding the random noise to the original LWIR images, with an average of 86.3 percent they confirm that LBP method decreases its performance in the presence of this kind of noise.

\subsection{The Glasses Present Case}

It is well known that in the presence of glasses the performance of the recognition algorithms for thermal images is very low [5. There is some work addressing this problem, e.g. in [6] where they detect and remove the eyeglass area in the 
Table 4. Classification results with the LBP method adding random noise

\begin{tabular}{|c|c|c|c|c|c|c|}
\hline & VA & EA & VF & EF & VL & EL \\
\hline VA & & 90.26 & 86.64 & 86.77 & 92.26 & 90.01 \\
\hline EA & 87.03 & & 81.06 & 80.16 & 87.55 & 91.05 \\
\hline VF & & 82.27 & & 89.89 & 86.14 & 85.27 \\
\hline EF & 89.14 & & 88.76 & & 85.61 & 83.33 \\
\hline VL & & 89.01 & 83.02 & 82.77 & & 88.64 \\
\hline EL & 86.79 & & 80.50 & 82.64 & 87.92 & \\
\hline
\end{tabular}

LWIR images in order to improve the recognition performance. We conducted an experiment to test the performance of the LBP method in LWIR face images when people are wearing glasses.

In this case, we used the VA subset as a gallery set and constructed three new test sets using only the LWIR images of those people who are wearing glasses in the following manner:

GF: all glasses images with frontal illumination.

GL: all glasses images with left lateral illumination.

GR: all glasses images with right lateral illumination.

Table 5 shows the results of the LBP method in the presence of glasses, for the original images and also for the same set of images corrupted with additive random noise. As can be seen, in general, the performance of the method is lower than for the no glasses case. However the results are comparable and even better on average than those obtained with the earlier methods which need to detect and preprocess eyeglass regions. The method used in [6], for example, achieves a recognition rate of 85.77 percent. Once again it can be appreciated that the results are affected by random noise.

Table 5. Correct classification with LBP in the presence of glasses

\begin{tabular}{|c|c|c|c|c|}
\hline & GF & GL & GR & Average \\
\hline VA-original images & 83.67 & 85.41 & 89.58 & 86.20 \\
\hline VA-with added noise & 61.22 & 64.58 & 66.67 & 64.14 \\
\hline
\end{tabular}

\section{Conclusions}

This paper applies the Local Binary Pattern method to LWIR face imagery, in order to perform person identification tasks. We observed through experimentation that LBP performs well on LWIR images, as compared to the state of the art methods for these kind of images, having some advantages compared with other appearance based methods in its simplicity of implementation and not needing any training images. It is important to note that the good results were obtained although the images were miss-aligned, which is an important property for thermal face recognition where it is easy to detect the face region but not the face 
features to make a geometric normalisation. Even in the presence of glasses the LBP method achieves good results without applying any preprocessing step. On the other hand we note that a magnified random noise affects the identification rate of the LBP method and the application of NU compensation magnifies this kind of noise, although it reduces the fixed-pattern noise. In conclusion it can be said that the LBP method is robust to the fixed-pattern noise and it may be applied directly to the LWIR face imagery with an identification rate average of 97.3 percent, achievable on good quality images.

\section{References}

1. Zou, X., Kittler, J., Messer, K.: Illumination Invariant Face Recognition: A Survey. In: First IEEE International Conference on Biometrics: Theory, Applications, and Systems, pp. 1-8 (2007)

2. Zou, X., Kittler, J., Messer, K.: Face recognition using active near-ir illumination. In: British Machine Vision Conference Proceedings (2005)

3. Li, S.Z., Chu, R., Liao, S., Zhang, L.: Illumination invariant face recognition using near-infrared images. IEEE Trans. Pattern Anal. Mach. Intell. 29(4), 627-639 (2007)

4. Socolinsky, D.A., Wolff, L.B., Neuheisel, J.D., Eveland, C.K.: Illimination invariant face recognition using thermal infrared imagery. In: Proc. IEEE CS Conf. Comp. Vision and Pattern recognition, vol. 1, pp. 527-534 (2001)

5. Kong, S.G., Heo, J., Abidi, B.R., Ki Paik, J., Abidi, M.A.: Recent advances in visual and infrared face recognition - a review. Computer Vision and Image Undertanding 97(1), 103-135 (2005)

6. Heo, J., Savvides, M., Vijaya Kumar, V.K.: Performance Evaluation of Face Recognition using visual and thermal imagery with advanced correlation filters. In: Conference on Computer Vision and Pattern Recognition, pp. 9-14. IEEE Computer Society, Los Alamitos (2005)

7. Socolinsky, D.A., Selinger, A.: A Comparative Analysis of Face Recognition Performance with Visible and Thermal Infrared Imagery. In: ICPR 2002: Proceedings of the 16Th International Conference on Pattern Recognition, vol. 4(4) (2002)

8. Bebis, G., Gyaourova, A., Singh, A., Pavlidis, I.: Face recognition by fusing thermal infrared and visible imagery. Image and Vision Computing 24(7), 727-742 (2006)

9. Singh, R., Vatsa, M., Noore, A.: Integrated multilevel image fusion and match score fusion of visible and infrared face images for robust face recognition. Pattern Recogn. 41(3), 880-893 (2008)

10. Chen, X., Flynn, P.J., Bowyer, K.w.: PCA-based face recognition in infrared imagery: baseline and comparative studies. In: IEEE International Workshop on Analysis and Modeling of Faces and Gestures, AMFG, pp. 127-134 (2003)

11. Pron, H., Menanteau, W., Bissieux, C., Beaudoin, J.L.: Characterization of a focal plane array (FPA) infrared camera. Quantitativa Infrared Thermography QIRT Open Archives, QIRT, 2000-2061 (2000), http://qirt.gel.ulaval.ca

12. Milton, A., Barone, F., Kruer, M.: Influence of nonuniformity on infrared focal plane array performance. Opctical Engineering 24, 855-862 (1985)

13. Hardie, R., Hayat, M., Armstrong, E., Yasuda, B.: Scene-based nonuniformity correction using video sequences and registration. Applied Optics 39, 1241-1250 (2000)

14. Ratliff, B., Hayat, M., Tyo, J.: Generalized algebraic scene-based nonuniformity correction algorihtm. The JOSA-A Opt. Soc. of America 22, 239-249 (2005) 
15. Harris, J., Chiang, Y.: Nonuniformity correction of infrared image sequences using constant statistics constraint. IEEE Trans. on Image Processing 8, 1148-1151 (1999)

16. Pezoa, J., Hayat, M., Torres, S., Rahman, M.: Multimodel kalman filtering for adaptive nonuniformity correction in infrared sensors. The JOSA-A Opt. Soc. of America 23, 1282-1291 (2006)

17. Averbuch, A., Liron, G., Bobrovsky, B.Z.: Scene based non-uniformity correction in thermal images using Kalman filter. Image and Vision Computing 25, 833-851 (2007)

18. San Martin, C., Meza, P., Torres, S., Carrillo, R.: Improved Infrared Face Identification Performance Using Nonuniformity Correction Techniques. In: Blanc-Talon, J., Bourennane, S., Philips, W., Popescu, D., Scheunders, P. (eds.) ACIVS 2008. LNCS, vol. 5259, pp. 1115-1123. Springer, Heidelberg (2008)

19. Ahonen, T., Hadid, A., Pietikäinen, M.: Face recognition with local binary patterns. In: Pajdla, T., Matas, J. (eds.) ECCV 2004. LNCS, vol. 3021, pp. 469-481. Springer, Heidelberg (2004)

20. Marcel, S., Rodriguez, Y., Heusch, G.: On the Recent Use of Local Binary Patterns for Face Authentication. International Journal on Image and Video Processing Special Issue on Facial Image Processing (2007)

21. http://www . equinoxsensors.com/products/HID.html

22. Belhumeur, P.N., Hespanha, J.P., Kriegman, D.J.: Eigenfaces vs. Fisherfaces: Recognition using class specific linear projection. IEEE Trans. Pattern Anal. Machine Intell. 7(19), 711-720 (1997) 